\title{
Granular Materials and the Risks They Pose for Success on the Moon and Mars
}

\author{
R. Allen Wilkinson ${ }^{1}$, Robert P. Behringer ${ }^{2}$, James T. Jenkins ${ }^{3 a}$, Michel Y. \\ Louge $^{3 \mathrm{~b}}$
}

\author{
'Microgravity Fluid Physics Branch, NASA Glenn Research Center, Cleveland, OH 44135, USA \\ ${ }^{2}$ Department of Physics and Center for Nonlinear and Complex Systems, Duke University, Durham, NC \\ 27708, USA \\ ${ }^{3 a}$ Department of Theoretical and Applied Mechanics, Cornell University, Ithaca, NY 14853, USA \\ ${ }^{3 b}$ Department of Mechanical and Aerospace Engineering, Cornell University, Ithaca, NY 14853, USA \\ '216-433-2075, Allen.Wilkinson@grc.nasa.gov
}

\begin{abstract}
Working with soil, sand, powders, ores, cement and sintered bricks, excavating, grading construction sites, driving off-road, transporting granules in chutes and pipes, sifting gravel, separating solids from gases, and using hoppers are so routine that it seems straightforward to do it on the Moon and Mars as we do it on Earth. This paper brings to the fore how little these processes are understood and the millennialong trial-and-error practices that lead to today's massive over-design, high failure rate, and extensive incremental scaling up of industrial processes because of the inadequate predictive tools for design. We present a number of pragmatic scenarios where granular materials play a role, the risks involved, and what understanding is needed to greatly reduce the risks.
\end{abstract}

\section{INTRODUCTION}

Granular physics is still very young and granular engineering does not yet have a firm predictive basis. As a consequence, many practical engineering solutions in industry depend on models that are not supported by basic physical understanding and experiment. Such practical devices do work on average, but granular devices are notorious for failing, often with destructive or fatal results, and at rates that far exceed fluids handling devices (Eibl, 1984). Ad hoc fixes and significant over-design are fairly routine. A Rand study (Rand Corp., 1986) found that granular devices operate at only 63\% of design capability. Seemingly modest changes in conditions, such as temperature, humidity, and surface conditions routinely cause earth-bound devices to fail. Granular engineering can only claim to be approximately predictive. Those setting design standards across the world (e.g. Prof. Michael Rotter, Univ. of Edinborough, who are actively engaged in setting European standards for granular devices) increasingly recognize that explicit predictive design criteria and correlations are woefully inadequate and that much research is needed to make cost effective designs and scale-up choices on Earth.

The current level of understanding is inadequate to design devices that must work without fail in environments that are hostile and poorly characterized, as they are in extraterrestrial locations. In contrast to damp terrestrial soils, Lunar and Martian soils are dry and charge electrostatically. Lower gravity and different particle shapes, and, perhaps, density can lead to porosities of excavated soils and graded surfaces that differ from those on Earth and are likely to reduce the load-bearing capacity of the soil. The "Lunar Sourcebook" (Heiken, 1991) contains extensive data on the numerous soil samples returned from the Moon. However, its characterization of soil does not take into account the research strides in granular physics that began in the very late 1980's. It is not mindful of the role of the granular jamming transition at a distinct soil porosity and the very inhomogeneous structure of force distributions in granular systems. The use of pneumatic transport of granular regolith to avoid dust creation will lead to jamming problems and clustering 
instabilities that are common on Earth. In order to avoid excessive EVA requirements, it is necessary to understand how to prevent or manage such jamming, and what role gravity plays in the formation of clusters (Griffith and Louge, 1998). The creation of blast craters and the dispersal of the resulting granular sediment associated with landing the more massive spacecrafts of the future are not sufficiently understood to prevent difficult or dangerous landings on uneven surfaces or unstable soils, or the damage to spacecraft due to flying granular debris. The potential for major catastrophe is high if human lives will depend on the granular behavior of extraterrestrial soils and regolith.

It is not possible to simply rescale earthbound designs to work elsewhere in the solar system, because such scaling does not work for granular devices on the Earth. Earth-based granular processing plants are built after unusually extensive precursor process and scaling-up trials. Design engineers are loathe to assume changes of conditions like humidity, gas pressure, and particle morphology, let alone gravity, are within the operating range of their plant unless they are explicitly tested. This leads to considerable iterative testing under the exact conditions and at the actual scale of production. This approach, which is affordable on Earth, is not affordable by NASA for the Moon and Mars. If more predictive design tools were developed for granular transport, sedimentation, and packing, then testing and scale-up costs could be greatly reduced.

There is much that must be learned about the science of granular materials if NASA is to have successful long-term explorations of the Moon and Mars. On a positive note, there has been truly exceptional interest in these materials within the past few years. New ideas abound, and real advances are being made in the modeling, experimental testing and the numerical simulation of the behavior of granular materials. It is reasonable to hope that our understanding of granular materials will indeed be sufficiently advanced by the time of the proposed flights that truly reliable devices will be possible. This can only be accomplished by sustained support for understanding the basic science of granular materials.

We have organized this paper in three categories of granular issues: 1) Transport, 2) Segregation, and 3) Structural Failure. In each category we will start with describing an operational scenario, identify the granular phenomena and its risks or unknowns, followed by what research is underway or is needed to acceptably minimize the risks.

\section{TRANSPORT}

\section{Pneumatic Transport}

In-situ Resource Utilization (ISRU) will involve moving granular powders or agglomerates from mine to plant or within a plant by pneumatic transport in closed pipes. Because the drag force on agitated aggregates of particles is not well characterized and the mechanisms by which energy is lost are poorly understood, it is impossible to design processing equipment to eliminate jamming in dense flows. Other technical unknowns of jams are the volume fraction of onset for any particulate system and the force variations to expect in the jammed state. Jammed particles interrupt the process until the jam can be cleared and the process of jamming may damage pipes and pumps. While jamming is undesirable in granular transport, we note later that for the stability of soils, jamming is desirable. The human intervention that is a common Earth-based solution to fix jammed systems is expensive, slow, and dangerous on the Moon and Mars.

Upon scale-up to realistic plant size, pneumatic transport is known to develop clustering instabilities that can lower the average drag on the particulate suspension by an order or magnitude from that of a homogeneous suspension of the same mean concentration (Grace, et al, 1997; Agrawal, et al 2001). The clusters can also merge into "ropes" that concentrate the particles within narrow bands aligned with the flow. Such ropes make it very difficult to predict the behavior of passive gas-solid separators such as cyclones (Bricout and Louge, 2004a), which are used downstream of a transport line or reactor to extract clean gaseous products from the suspension. The interactions of suspended solids with boundaries also affect the overall flow behavior, such as the descent of clusters at the wall of a circulating fluidized bed (Grace, et al, 1997), and can cause extensive damage to surfaces by wear. All such phenomena depend on gravity, particle size, particle shape, electrostatics, environmental conditions and unit size in ways that are not yet predictable without extensive tests on realistic pilot plants. 


\section{Emptying Storage Hoppers}

On Mars, mined regolith must be stored in some fashion, and this will probably involve hoppers, bins, etc. However, even on Earth, the emptying of hoppers, bins, or other containers is fraught with uncertainty and danger. Structural failure of hoppers due to instabilities and fluctuations during emptying is all too common. Indeed, Eibl, estimates that (on Earth) the failure rate of granular handling devices is 100 to 1000 times that of fluid-handling devices. The causes for these failures are both complex and unknown. However, a key ingredient is that pressure distributions after filling can vary significantly from one nominally identical filling to another as a result of the complex rheology associated with granular flows. A probabilistic description of such processes is now becoming a part of standard engineering practice (Rotter, 2003). The need for a probabilistic description is likely fundamental, and has to do with the strongly fluctuating character of granular flow at a most fundamental level. The possible range of flow behavior for even simple materials is an important and yet-to-be-determined aspect of ongoing research. Single tests of materials may well not establish a range of possible properties and behaviors and devices that are designed for one geometry and/or material cannot be used safely for others. Before handling devices are developed for Martian or Lunar environments, there is a crucial need for careful materials characterization. In this regard, standard engineering tests, such as Jenike shear cell tests are likely to be inadequate, as indeed, they are frequently inadequate on Earth.

\section{Excavation}

Excavation of regolith will be common for raw material, for above surface construction, and for sub-surface radiation protected habitats. The existence of massive avalanches on Mars indicates that the stability of slopes and the flows down slopes may be different on Mars from those on Earth. In fact, recent work suggests that the origin of Martian gullies may be related to dry granular flows unknown on Earth, rather than ancient entrainment of water-saturated debris flows (Shinbrot, et al, 2004; Treiman, 2003). At the least, such debate betrays a lack of consensus on the underlying mechanisms for powder mechanics on Mars. As for storage containers, the controlling material properties must be carefully determined. Again, a range of possible behavior is likely to be observed, and this range must be accounted for. Materials handling and material flowability will be crucial issues and must be carefully considered in advance for a given setting. Numerical modeling and experimental testing to determine the range of possible behavior will provide important insights.

Because the hydrodynamics and reactivity of gas-solid reactors crucially depends on achieving an optimum particle size distribution, comminution, sifting, separation and beneficiation closely follows excavation. Such processes are among the most energy-inefficient industrial operations on Earth. In addition, attrition, breakage, fracture, and sieving are highly powder-dependent, and will require realistic samples before designing an optimum extra-terrestrial process. Again, numerical modeling and experiment will be crucial to determine the range of possible behavior.

\section{Rocket Landing Blast/Scouring Zones}

Rockets for Lunar and Martian landing will be much larger than on the Appollo Lunar lander. These rockets, during landing, will scour the surface, modify the shape and composition of the landing area and, by sending particles flying far and wide, can damage spacecraft. Extreme local modifications of the surface could also prohibit a safe landing. Prediction of scouring requires a better understanding of the interaction between the hot gases of the exhaust and the flowing granular aggregate. In addition, the soil blasted away during landing and takeoff will deposit around the craft and settle to a porous state compared to virgin soil. This may be managed with launch pads which can be constructed after some number of initial risky landings. More will be said about disturbed soils and their uncertain properties in the Structural Failure section.

\section{Dust Settling}

Mars is a dusty environment, and there are a number of significant issues in this regard that must be addressed. Dust storms are common and often suspend great quantities of material. The Moon experiences 
UV electrostatic charging of dust and its levitation in the dawn/dusk boundaries. Astronauts noted lunar dust quickly attached itself to boots, suits and tools on first contact with the soil. Human and robotic activity will stir up dust on the Moon and Mars. Dust will likely have much different levitation times with different particle size and morphology distributions than on Earth due to lower gravity, anhydrous ambients, and the mechanical history of the soils. Mars will differ form the Moon given Mars' atmosphere. Explosion hazards are possible from the fine reactive Martian dust. Human habitat, health and clothing, as well as support equipment, such as solar panels, bearings and respirators, are vulnerable to dust. Consequently, it is important to understand how dust is suspended and transported and how it settles after being disturbed. Van der Waals and electrostatic surface forces are expected to play roles. However, these forces are difficult to assess without resorting to empirical tests (Feng and Hays, 2003; Colver, 2000).

\section{Fluidized Bed Processes}

Fluidized beds are likely to be used on the Moon and Mars in processes to extract useful gases from the regolith. These gases may well include such essentials as water (vapor), hydrogen, and oxygen. Fluidized beds are notoriously difficult to design for applications on Earth and, so far, have resisted efforts to infer the behavior of a full-scale system from that of a prototype. Fluidized beds can also be subject to overall dynamic instabilities, in which the entire plant resonates toward failure in ways that are only understood in the simplest of situations (Mountziaris and Jackson, 1991; Jackson, 2000). Fortunately, experimental techniques can reveal the role of scale-up, pressure and gravity using dimensional similitude (Bricout and Louge, 2004b), as long as basic particle properties like material density and size distribution are known.

\section{SEGREGATION}

\section{Segregation in Solid-Fluid Mixtures}

Exhaust gases of space vehicles will scatter granular soil and regolith according to their size and mass. The creation of safe landing area will require an understanding of how granular materials are separated by size and mass when interacting with hot turbulent gases and colliding with one and other. Slurries, such as concrete, that contain regolith particles of different sizes may be employed as construction materials. An inhomogeneous distribution of particles in concrete may lead to its failure. Consequently, it is important to understand how the size distribution of the particles in an aggregate influence their position in the flow.

\section{Sifting and Sorting Excavated Regolith}

Excavated regolith will have to be sorted by size to facilitate cements mixing, reactor bed construction, and ore mining. It will likely be important in laying road beds as it is on Earth. On Earth, mineral processing devices such as pinched sluices, Humphreys spirals, and Reichert cones are employed for sorting materials of different sizes and different densities (Wills, 1979). Such devices take advantage of gravity separation when dry granular materials flow down an inclined surface. However, despite the fact that they have been used for centuries, the mechanics of these devices is poorly understood. The sorting could also take place by shaking, with sieves sometimes involved. An understanding of how segregation by size takes place in granular flows and vibrated beds in weak gravity fields is just beginning to be developed. With sieves, the issues of flow in pipes and hoppers noted earlier are also important.

\section{Preparing Powder Mixes for Sintered and Pressed Materials}

Sintered and pressed materials can be produced in low or zero gravity. However, for greatest strength, they must consist of a mixture of particles of different sizes that are homogeneously distributed throughout the material. Consequently, the different diameter and shaped particles must be well mixed. However, an understanding of how particles of different diameters mix in zero or low gravity is just beginning to be developed. 
In addition, there is the unknown of the natural packing density and compaction time constants of these materials in partial gravity. If the network of contacting particles is not dense enough, then the material will be unusually weak. On the other hand, these same extra porous materials may be better than usual thermal insulators.

\section{STRUCTURAL FAILURE}

\section{Trafficability on Virgin Sands and Graded or Excavated Soils}

A crucial aspect of exploration will involve the reliable mobility of rovers and humans over the Martian surface. It will be a challenge to do this safely under all circumstances. Already, during the present Mars mission, there have been some close calls for NASA vehicles, and it may be that the Beagle was stranded inside a crater or was buried in soft sand. There is a crucial need to develop devices that can assess the stability of a surface before a load from a person or a vehicle is placed on it. Similarly, a significant effort should be made in the design of vehicles that can adapt to different surface conditions to avoid becoming stranded.

On Earth, water flow and gravity have compacted most soils. However, trafficability on disturbed Lunar and Martian soils on graded road beds and backfilled construction and mining sites is not explored. Such soils may not support loads well. The worst case scenario would be soil behavior similar to quick-sand or snow with loss of life and equipment.

Figure 1: This image shows the photo-elastic response of a model 'vehicle', in this case a kind of sled, that is being propelled across a special substrate that consists of photo-elastic particles. Force chains are clearly visible and occur in response to two effects: 1) gravity, and 2) stresses that are set up in response to the shear as the sled is pulled across the surface. As the sled advances, these chains fluctuate strongly. We expect that similar structures exist under real vehicles. WEX OK st

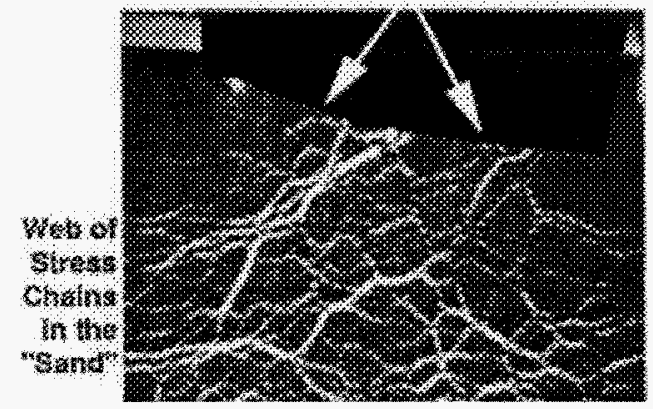

The reason that compact soils are stable is that there are rigid networks of contacts. These networks lead to filamentary structures called stress chains. And, the majority of the force is then carried on these chains. When a granular system expands too much, these chains are broken, and its load-carrying ability vanishes. It is perhaps surprising that the change in solids fraction needed to go from rigid to weak, a process known as unjamming, is only a few percent. Conversely, a small amount of compaction can jam a loose soil. Loads on granular soils induce flow away from the high stress areas as fluids do. Hence, rheology plays role, which is poorly understood as well. Current research associated with the GFM studies the force chain network in granular media. Fig. 1 reflects the inhomogeneity of the network given a local load like a foot or a wheel. It is hard to think of soils as continuous plastic and elastic media with this picture in mind, and current research is directed at developing new ways to understand the nature of granular solids and jamming.

\section{Traction for Hill Climbing and Excavating}

In addition to static stability of surfaces, vehicles must be able to apply shear dynamically to the soil. Vehicles must be able to adapt so as to provide traction on steep slopes and embankments that may be close to the point of avalanching. Indeed, some method of determining the stability of slopes should be developed so that vehicles do not inadvertently venture up or down an unstable slope.

Excavation is another important process for Lunar/Martial missions. One common excavation scenario consists of swarms of robots each with a front loader or toothed blade digging into virgin soil, scooping it up, and possibly carrying it up onto a dump pile. Later, these piles might be used for hauling to a processing 
plant or backfilling a habitat construction. We are unaware of quantitative work on the drawbar force and power needed to dig or loosen virgin Lunar or Martian soils. This then translates into a required dynamic shear force applied to the soil by a wheel or track. If a particular path of the robots is frequented, the soil in the path is no longer virgin and may exhibit unexpected instabilities due to lower shear and compressive strength. Driving on the dump pile or the habitat backfill area raises problems discussed in the previous subsection.

\section{Buildings on Virgin and Excavated Soils}

The Lunar Sourcebook characterizes the virgin Lunar soil as essentially robust for buildings and landing. The eons of seismic activity and meteorite impacts have created a well compacted regolith in many places. It does, however, caution about the local variability of allowable bearing capacity and the effect on structures like telescopes that require good stability against settling. The book also makes some cautionary notes about vibratory (seismic or otherwise) and diurnal temperature swings on soil bearing capacity and building stability.

There is no direct discussion of building stability on excavated soils. With repeated mining and other human activities more and more of the Lunar surface will cease to be virgin. Virgin soil void ratios are noted from 0.67 to 2.37 . This implies granular solids volume fractions from $80 \%$ to $30 \%$ respectively. A void ratio of one corresponds to solid volume fraction of $50 \%$. In simple granular systems the jamming transition, that is the physical basis for soil load bearing capacity, occurs for a solids volume fraction of about 0.63 . A decrease of the solids fraction by only a small amount, say 0.05 , means that it no longer can support loads. The characterization of the jamming transition is currently an active area of research that needs support.

\section{RESEARCH TO ADDRESS TRANSPORT, SEGREGATION, AND STRUCTURAL FAILURE}

\section{Transport}

The necessary research involves the study of two important aspects of gas-solid flows over a range of flow conditions: the characterization of the drag exerted by the gas on agitated particles, and the determination of the amount of energy transferred between the gas and the particles. This will permit the rational design of pneumatic-transport lines and fluidized beds and permit a description of Aeolian transport of sand and the settling of dust on Mars. Experiments measuring gas drag and the viscous dissipation of particle agitation energy over a relatively small range of carefully controlled flow conditions are planned for the Granular Flow Module (GFM) in the International Space Station (ISS). Additional experiments over a wider range of flow parameters should be carried out. It is also important to understand the phenomena of clustering and jamming in gas-particle systems. Pneumatic transport fails by jamming and granular beds must be unjammed before being fluidized. These phenomena will also be included in a study of granular rheology, fluctuations, and transitions in the GFM that is described in more detail below.

Numerical simulations using the Lattice-Boltzmann (LB) technique (Ladd and Verberg, 2001; Xu, et al, 2004) will soon be able to capture two of the three GFM experiments. Discrete element simulations can capture much of the behavior of the third GFM experiment. Those experiments will serve as a well-controlled tool to validate the simulations. Once validated, the LB simulations can be used to establish constitutive relations for gas-solid suspension involving realistic particle shapes and size distribution. In turn, the constitutive relations can be used to create models of larger systems for practical applications.

\section{Segregation}

The necessary research on segregation and mixing in granular systems must focus on mechanisms of segregation that are important in Mars and Lunar gravity. In low gravity, a mechanism of segregation that is 
associated with collisions between particles becomes as, or more, important than gravitational mechanisms. This mechanism has been studied on the $\mathrm{KC}-135$ and in planned experiments in the GFM on the ISS. An understanding of collisional segregation will contribute to the design of processes in the mining and refining of regolith and will contribute to the selection and construction of landing sites. In particulate flows that involve gases and fluids, segregation can be driven by a differential drag of particles with different size and masses and influenced by their collisional interactions. Segregation in such flows must be better understood in order for particle separation processes in low gravity that involve flows of liquids and gases to be effectively designed and for ejecta and structure of areas exposed to exhaust gases to be predicted.

The study of segregation includes the study of dry granular flows as they are encountered in, for example, avalanches. Methods from the kinetic theory (e.g. Jenkins, 1999) have provided models for granular flow and segregation that are predictive or, at least, suggestive. Because reduced gravity, granular flows on the Moon and Mars are likely to involve collisions between particles rather than enduring contact, such flow models are likely to be relevant. Numerical simulations of aggregates of discrete particles have been shown (Louge, et al, 2003) to predict the observed flow features and concentration profiles in segregating flows. This numerical tool has the potential to assist in the design of materials handling devices, but more extensive testing of it should be carried out.

\section{Structural failure}

The necessary research in this area involves the characterization of statistical properties of granular materials, the determination of rheological properties, and the prediction of transitions between solid and fluid-like granular states. Work currently in progress and scheduled to fly on the ISS as part of the GFM will take significant steps towards characterizing the rheology and stability of granular materials under highly controlled conditions. Work that is covered under GFM will probe the rheology of a broad range of states, and has the ability to provide the key physics that could determine the stability of solid-like granular surfaces and their likely behavior if they fail and start to slip due to applied shearing loads. Specifically, this work is focused on the nature of the fluid-solid granular transition, the stresses that are typical of each state, and the range of behavior that can be anticipated in each state. It is noteworthy that the kind of information that these studies would yield is a) not currently available, b) is obtainable only under conditions of essentially 0 -g, and c) would be invaluable in the design of any number of devices, as noted above. The ongoing research on the structural properties of granular materials may well help to identify techniques for detecting instability before a load is applied, or even possibly developing methods to help stabilize soil that is close to failure.

For example, in the robot swarms excavation example in this paper, research on force chains and the jamming transition will provide the sound physical basis to predict drawbar force and power; the shear strength of soils for traction on embankments and lose fill piles; and the load bearing capacity of lose fill piles.

\section{CONCLUSIONS}

NASA has grown to depend on the Lunar Sourcebook to understand lunar soil mechanics. It was written when current research into granular materials was re-awakening. NASA has an opportunity to make a lasting contribution in his field while improving the reliability and reduce costs of extraterrestrial operations. It could even save lives by knowing better when not to drive or walk on piles or near steep slopes.

An understanding of the behavior of granular materials will be a key to the successful exploration of the Moon and Mars, which are made up largely of granular materials. We have indicated examples of situations and processes in which granular materials will play an important role in exploration activities. At present, there is not a sufficient base of knowledge to permit rational prediction of the response of granular materials in such situations. Consequently, costly over-design and testing are required, without guarantee that safe performance will be attained over a range of operating conditions. We have outlined what research is necessary to improve upon this situation and what research is already planned for applications in the low gravity of the Moon and Mars. 


\section{REFERENCES}

Committee on Microgravity Research, Space Studies Board, Commission on Physical Sciences, Mathematics, and Applications, National Research Council, Microgravity Research in Support of Technologies for the Human Exploration and Development of Space and Planetary Bodies, National Academy Press, Washington, D.C., 2000

Bricout,V. and M. Louge, M., "Measurements of cyclone performance under conditions analogous to pressurized circulating fluidization," Chem. Eng. Sci. 59, 3059-3070 (2004a)

Bricout, V. and Louge, M. "A verification of Glicksman's reduced scaling in pressurized circulating fluidization," Chem. Eng. Sci. 59, 2633-2638 (2004b).

Colver, G. M., "An Interparticle Force Model for ac-de Electric Fields in Powders," Powder Tech. 112, 126-136 (2000).

Eckart P., The Lunar Base Handbook, Space Technology Series, McGraw Hill, 1999.

Eibl, J., "Design of silos --- pressures and explosions”, The Structural Engineer, 62A, 169ff (1984)

Feng, J. Q. and Hays, D. A., "Relative importance of electrostatic forces on powder particles," Powder Tech. 135, 65-75 (2003).

Grace, J., Knowlton, T. and Avidan, A., Circulating Fluidized Beds, Blackie Academic \& Professional, (1997).

Griffith, E. and Louge, M. "The Scaling of Cluster Velocity at the Wall of Circulating Fluidized Bed Risers," Chem. Eng. Sci. 53, 2475-2477 (1998).

Heiken G.H., Vaniman D.T., and French B.M., Lunar Sourcebook: a user's guide to the Moon, Cambridge Univ. Press, Cambridge, 1991

Jackson R., Dynamics of Fluidized Particles, Cambridge Univ. Press, Cambridge, MA (2000)

Jenkins, J. T., "Kinetic theory for nearly elastic spheres” In Physics of Dry Granular Media (Herrman, H. J., Hovi, J.-P., and Luding, S., Eds,) 353-370, Kluwer: Dordrecht (1999).

Ladd, A. J. C. and Verberg, R., "Lattice-Boltzmann simulations of particle-fluid suspensions," J. Stat. Phys. 104, 1191$1251(2001)$.

Louge, M., Xu, H. and Reeves, A.,"Solutions of the Kinetic Theory for Bounded Collisional Granular Flows," Continuum Mechanics and Thermodynamics 15, $321-349$ (2003).

Mountziaris, T. J. and Jackson, R., "The effects of aeration on the gravity flow of particles and gas in vertical standpipes," Chem. Eng. Sci. 46, 381-407 (1991).

Rand Corp. Study, Rept. R-3216-DOE/PSSP, 1986

Rotter, M., Personal communication (2003).

Shinbrot, T., Duong, N.-H., Kwan, L. and Alvarez, M. M., "Dry granular flows can generate surface features resembling those seen in Martian gullies," PNAS 101, 8542-8546 (2004).

Agrawal K, Loezos PN, Syamlal M, and Sundaresan, S., "The Role of Meso-scale Structures in Rapid Gas-Solid Flows.” J. Fluid Mech. 455, 151-185 (2001).

Treiman A.H., "Geologic settings of Martian gullies: Implications for their origins." J. Geophys. Res. 108, DOI 10.1029/2002JE001900.

Wills, B. A., Mineral Processing Technology. Pergamon (1979).

Xu, H., Verberg, R., Koch, D. L. and Louge, M. Y., "Dense bounded shear flows of agitated solid spheres in a gas at intermediate Stokes and finite Reynolds numbers," J. Fluid Mech. (2004), under review. 